\title{
Autosomal Dominant Inheritance
}

National Cancer Institute ( $\mathrm{NCl})$

\section{Source}

National Cancer Institute (NCI). Autosomal dominant inheritance.

Autosomal dominant inheritance refers to genetic conditions that occur when a mutation

is present in one copy of a given gene (i.e., the person is heterozygous). 